\title{
Endothelium-targeted human Delta-like 1 enhances the regeneration and homing of human cord blood stem and progenitor cells
}

\author{
Deng-Mei Tian' ${ }^{1}$ Ying-Min Liang ${ }^{2}$ and Yong-Qing Zhang ${ }^{1 *}$
}

\begin{abstract}
Background: Umbilical cord blood (UCB) is becoming an alternative cell source for hematopoietic stem cell transplantation (HSCT). However, umbilical cord blood transplantation (UCBT) has been severely limited by low and finite numbers of hematopoietic stem cells and their delayed engraftment. New strategies are needed to improve ex vivo expansion efficiency and in vivo haematopoietic recovery.

Methods: We produced an endothelium-targeted soluble Notch ligand, the Delta-Serrate-Lag-2 (DSL) domain of human Delta-like 1 fused with a RGD motif ( $h D 1 R)$, and tested the effects of this protein on human umbilical cord blood hematopoietic stem and progenitor cell (UCB-HSPC) ex vivo and in vivo.

Results: hD1R-mediated ex vivo expansion system was able to significantly increase the absolute number of UCBHSPCs. The hD1R-expanded cells had the enhanced homing and maintained long-term hematopoietic stem cell repopulation capacity in the bone marrow of immunodeficient nonobese diabetic-severe combined immunodeficient (NOD/SCID) mice. Moreover, systemic administration of hD1R promoted the in vivo regeneration of donor cells in recipient mice and accelerated hematopoietic recovery, particularly in settings wherein the HSPCs dose was limiting.
\end{abstract}

Conclusions: Our results indicated that hD1R might be applied in improving hematopoietic recovery and HSC engraftment in human UCBT.

Keywords: HSPCS, Notch, Delta-like 1, Regeneration, Homing

\section{Background}

Allogeneic hematopoietic stem cell transplantation (Allo-HSCT) is recognized as an effective treatment for patients with hematological and nonhematological malignancies. Umbilical cord blood (UCB) is becoming an alternative source of hematopoietic stem cells for patients requiring allo-HSCT when no suitable donors are available [1]. Its rapid availability and tolerance to higher HLA disparity have more advantage than bone marrow (BM) or peripheral-blood (PB) stem cell transplantation. Despite these, the clinical application of UCB in the adults has been restricted by the low and finite

\footnotetext{
*Correspondence: zhangyongqing0725@163.com

1 Department of Hematology, 309th Hospital, Chinese People's Liberation

Army, Hei-san hu Street \#17, 100091 Beijing, China

Full list of author information is available at the end of the article
}

hematopoietic stem and progenitors cell (HSPC) dose, which resulted in delayed engraftment and increased rates of infectious complications [2, 3]. Several strategies were explored to ameliorate outcomes after UCB transplantation (UCBT), including infusion of double UCB units (DUCB), ex vivo expansion of HSCs and so on [4, 5]. Although DUCB increased HSPC numbers and promoted engraftment rates, the risk of GVHD (graft versus host disease) significantly rose [6]. In addition, multiple cytokines were used to expand HSPCs ex vivo in UCB, but few increased cells achieved long-term engraftment, suggesting expansion of hematopoietic progenitor cells (HPCs) [7, 8].

HSCs are normally accommodated in stem cell niches, where specific niche cells are thought to provide the necessary paracrine factors and direct cell-cell interaction 
to maintain the proliferation and differentiation of HSCs [9-11]. Many studies investigated the expansion of HSCs on mesenchymal progenitor, endothelial cell (EC) feeder layers, and $\mathrm{CD} 146^{+}$pericytes, which were used as surrogates for in vivo stem cell niches and provided necessary signaling cues to maintain the stemness of expanded cells [12-14]. However, due to difference in the cytokines used and loss of long-term engraftable cells in vitro expansion, an effective protocol for the expansion of UCB HSCs is still to be explored.

The Notch signaling pathway plays a key role in regulating the proliferation and differentiation of stem cells [15]. In mammals, the canonical Notch pathway includes five Notch ligands (Delta-like [Dll] 1, 3, 4, Jagged 1, 2), four Notch receptors (Notch1-4), the transcription factor RBP-J, and the downstream effectors such as the Hairy and enhancer of split (Hes) family molecules [16]. Early work showed that Notch receptors and ligands were expressed by BM stromal and hematopoietic cells [17, 18]. Targeted disruption of Notch pathway diminished stemness of HSCs. Several reports showed that Notch 1, Dll-1, and Jagged-1 could induce expansion of HSPCs, and that ligand immobilization was required to activate Notch signaling [19-22]. Other studies demonstrated the selectivity and dose-dependency of ligands in HSPCs expansion ex vivo [23]. Furthermore, Delaney et al. [24] reported that the average time of neutropenia was significantly shortened in patients who received human UCB $\mathrm{CD}_{4}{ }^{+}$cells expanded with the immobilized Delta 1 in a phase I clinical trial of transplantation.

Our previous research demonstrated endotheliumtargeted Delta-like 1 (D1R) significantly promoted the expansion of mouse and human HSPCs. Moreover, mouse D1R (mD1R) could directly stimulate hematopoiesis in vivo in normal or sublethally irradiated mice. mD1R might promote HSCs engraftment through influencing, structurally and/or functionally, stem cell niches [25]. However, the in vivo roles of human D1R (hD1R) on hematologic recovery and the engraftment of donor cells in HSCT were not explored in detail. In the present study, we purified $\mathrm{hD1}$ R fusion protein, which was composed of the DSL domain of the human Dll1 and a RGD (arginine-glycine-aspartic acid) motif binding to the endothelial integrin $\alpha \mathrm{v} \beta 3$ and triggering ligand endocytosis. We demonstrated that $\mathrm{hD} 1 \mathrm{R}$ promoted the regeneration and homing of human UCB HSPCs and enhanced HSC engraftment in vivo in transplantation.

\section{Methods}

\section{DNA recombination and production of recombinant} proteins

The cDNA fragment encoding the DSL domain (amino acids 127-225) of the human Dll1 (NM_005618) was amplified by PCR using a human cDNA library as a template. The product was fused at the $\mathrm{C}$-terminus with a RGD-encoding fragment (hD1R) by PCR. The resultant gene fragments were cloned into pET32a $(+)$ between the Nco I and Xho I sites, to construct pET32a-hD1R. For the production of recombinant proteins, E. coli BL21 (DE3) were transformed with the plasmids. Positive clones were expanded in Luria-Bertani (LB) medium, and cells at the exponential stage were induced with $0.5 \mathrm{mM}$ isopropyl $\beta$-D-thiogalactoside (IPTG). The Trx-tagged proteins were purified by using $\mathrm{Ni}^{+}$-NTA columns (Invitrogen, Carlsbad, CA) according to the manufacturer's manual. To obtain the S-tagged proteins, Trx-hD1R were cleaved by using thrombin (Novagen, Darmstadt, Germany), and further purified using $\mathrm{Ni}^{+}$-NTA columns. The hD1R protein was prepared in the Department of Medical Genetics and Developmental Biology of Fourth Military Medical University and has been detailed previously [25, 26].

\section{Cell culture}

Human umbilical vein endothelial cells (HUVECs) were cultured in M199 medium (GIBCO, Gaithersburg, MD) supplemented with $20 \%$ fetal bovine serum (FBS), $30 \mu \mathrm{g} /$ $\mathrm{mL}$ endothelial cell growth supplement (ECGS) (Sigma, St Louis, MO), 20 units $/ \mathrm{mL}$ heparin, $100 \mathrm{U} / \mathrm{mL}$ penicillin, and $100 \mu \mathrm{g} / \mathrm{mL}$ streptomycin. Cells between passage three and five were used for experiments. For co-culture, HUVECs $\left(2 \times 10^{4}\right)$ were seeded in wells of 24 -well plates and cultured to confluence. Cells were treated with mitomycin C $(10 \mu \mathrm{g} / \mathrm{mL})$ for $2.5 \mathrm{~h}$, and were washed with PBS thoroughly for three times. Human UCB CD34+ ${ }^{+}$progenitor cells were purified from human UCB samples by FACS-sorting after being stained with anti-human CD34-FITC (\#581, Biolegend). The cells $\left(2 \times 10^{3}\right)$ were then plated on HUVECs and cultured in serum-free medium (StemSpan SFEM, STEMCELL Technologies, Vancouver, Canada) supplemented with a cocktail containing five types of human cytokines (h5GF) including thrombopoietin (TPO, $20 \mathrm{ng} / \mathrm{mL}$ ), stem cell factor (SCF, $120 \mathrm{ng} / \mathrm{mL}$ ), Flt-3 ligand (Flt-3L, $50 \mathrm{ng} / \mathrm{mL}$ ), interleukin 6 (IL-6, $5 \mathrm{ng} / \mathrm{mL}$ ), and interleukin 3 (IL-3, $5 \mathrm{ng} / \mathrm{mL}$ ) (PeproTech, Rocky Hill, NJ). hD1R was added at the concentration of $2.5 \mu \mathrm{g} / \mathrm{mL}$ as previously described [25]. In some experiments, $\gamma$-secretase inhibitor (GSI) (DAPT, Alexis Biochemicals, San Diego, CA) was included at the concentration of $10 \mu \mathrm{M}$. Half amount of the medium was changed every other day. Seven days after the starting of the co-culture, cells in suspension were collected by gentle pipetting and analyzed further. In some experiments, confluent HUVECs were cultured for $48 \mathrm{~h}$ in serum-free medium and supernatant containing soluble factor were collected and filtered through a $0.22 \mu \mathrm{m}$ sterile filter as 
culture conditioned media. Live HUVECs were fixed $4 \%$ paraformaldehyde (PFA) for $15 \mathrm{~min}$ and then used for co-culture experiments. Experiments associated with human samples were approved by the Ethical Committee on Medical Research-Related Affairs of the Fourth Military Medical University.

\section{Colony-forming units (CFU) assay}

CFU assay was performed by mixing freshly isolated or cultured hematopoietic cells with Methocult GF H4434 medium (STEMCELL Technologies). Cells were cultured for 14 days, and colonies (with $>50$ cells) containing different lineages of cells were counted under a microscope.

\section{Flow cytometry}

FACS analysis was performed routinely by using a CaliburTM flow cytometer (BD Immunocytometry Systems). Anti-mouse CD45-FITC (\#104, eBioscience), anti-human CD45-APC (HI30, eBioscience), anti-human CD34-FITC (\#581, Biolegend). Cell-cycle analysis was performed using DNA binding dye propidiumiodide (PI). Hematopoietic cells were fixed in $50 \%$ ethanol and resuspended to $0.2 \mathrm{~mL}$ of $10 \mathrm{mg} / \mathrm{mL}$ RNAaseA and $50 \mu \mathrm{g} / \mathrm{mL}$ PI. Cellcycle kinetics was performed with routine protocols using the FACS Calibur flow cytometer (Becton-Dickinson, CA). Apoptosis was analyzed by using an Annexin V-FITC Apoptosis Detection Kit (4A Biotech, Beijing, China).

\section{Real time reverse transcription-polymerase chain reaction (RT-PCR)}

Total RNA was extracted by using the Trizol reagent (Invitrogen). cDNA was prepared by using a kit from TOYOBO (Osaka, Japan) with random primers. Real time PCR was performed by using a kit (SYBR Premix EX Taq, Takara) and the ABI Prism 7500 real time PCR system, with $\beta$-actin as a reference control. Primers used in RT-PCR were as follows: $\beta$-actin-F: 5'-TGGCACCCAGCACAATGAA; $\beta$-actin-R: 5 -CTAA GTCATAGTCCGCCTAGAAGCA; CXCR4-F: 5'-CCT ATGCAAGGCAGTCCATGT; CXCR4-R: 5'-CTAAGTC ATAGTCCGCCTAGAAGCA; Hes1-F: 5'-TGGAAATG ACAGTGAAGCACCTC; Hes1-R: 5'-TCGTTCATGCA CTCGCTGAAG; $\alpha 4$ integrin-F: 5'-GGAATATCCAGTT TTTACACAAAGG; $\alpha 4$ integrin-R: 5'-AGAGAGCCAG TCCAGTAAGATGA; $\alpha 6$ integrin-F: 5'-ATGCACGCGG ATCGAGTTT; $\alpha 6$ integrin-R: 5'-TTCCTGCTTCGTAT TAACATGCT.

\section{NOD/SCID transplantation}

NOD/SCID mice of 6-8 weeks old were purchased from Beijing HFK Bioscience Co. Ltd and were maintained in axenic conditions and sublethally (300 cGy) irradiated by total-body irradiation with $\gamma$-ray from a ${ }^{60} \mathrm{Co}$ irradiation apparatus. Freshly isolated BM cells or in vitro expanded cells were infused via the tail vein. Mice were then maintained with gentamycin sulfatecontaining water until further experiments. The homing efficiency of the transplanted cells was estimated by flow cytometry. For limit dilution assays, serial numbers of uncultured CD34 ${ }^{+}$UCB-derived cells or a fraction of the final culture equivalent to starting cells $\left(10^{3}, 6 \times 10^{3}, 10 \times 10^{3}\right.$ cells $)$ were transplanted. Positively engrafted mice were regarded as to have $\geq 0.5 \%$ of human $\mathrm{CD} 45^{+}\left(\mathrm{hCD} 45^{+}\right)$cell in peripheral blood 12 weeks after the transplantation. The percentage of the negatively engrafted mice was plotted against the initial numbers of cells infused. SCID-repopulating cell (SRC) frequency was estimated by using the L-Calc software (STEMCELL Technologies) as the cell number by which $37 \%$ of the mice were non-engrafted. For secondary engraftment, $50 \%$ of the bone marrow (from both femurs and tibiae) from each recipient mouse $\left(10 \times 10^{3}\right.$ cells group) was transplanted into one secondary sublethally irradiated mouse. 12 weeks after transplantation, bone marrow was harvested from the secondary mice and analyzed by flow cytometry. All animal experiments were approved by the Animal Experiment Administration Committee of the Fourth Military Medical University.

\section{Migration assays}

Chemotaxis experiments were performed in polycarbonate transwell inserts (5-mm pore, Corning Costar Corp.). Migration medium with $100 \mathrm{ng} / \mathrm{mL}$ SDF-1 (Peprotech) was added in the lower chamber. Freshly purified CD34 ${ }^{+}$ cells or cells expanded ex vivo $\left(2 \times 10^{5}\right)$ were seeded in the upper compartment. After $4 \mathrm{~h}$ of incubation at $37^{\circ} \mathrm{C}$, migrated cells in the lower chamber were enumerated by flow cytometry. The percentage of migration was calculated by dividing total cells migrated to the lower well by the cell input multiplied by 100 .

\section{Peripheral blood analysis}

Retro-orbital peripheral blood was collected using microhematocrit capillary tubes (Sanger Biotech, China). Differential blood counts were obtained using PE-6000 Hematology Analyzer (Prokan Electronics Inc., China).

\section{Histology}

Tissues were fixed in $4 \%$ paraformaldehyde overnight. Femurs were decalcified for 21 days using an EDTA decalcifying solution for paraffin section. H\&E staining of bone sections were performed using standard procedures. Images were taken under a fluorescence microscope (Olympus BX51, Japan). 


\section{Statistics}

Data were analyzed with the SPSS 17.0 software. Comparisons between groups were undertaken by using the unpaired Students' $t$ test. Results were expressed as the mean \pm standard deviation (SD). $P<0.05$ was considered statistically significant.

\section{Results \\ Direct cellular contact with HUVECs improved hD1R-induced human UCB HSPCs expansion ex vivo}

We purified a fusion protein composed of the DSL domain of human Dll1 and a RGD motif (hD1R) as previously described [25, 26] (Additional file 1: Figure S1A, $1 B)$. To assess the effects of hD1R on UCB HSPCs, we tried to expand human $\mathrm{CD}_{3}{ }^{+}$cells ex vivo under different culture conditions. It should be noted that different conditions were tested: (1) Co-culture with HUVECs supplemented with h5GF in the presence of PBS or hD1R; (2) Supernatant from HUVECs (sup group) in the presence of h5GF plus hD1R; (3) Fixed HUVECs by $4 \%$ PFA (fix group) with h5GF plus hD1R. Human cord blood CD34 ${ }^{+}$cells $\left(2 \times 10^{3}\right)$ were cultured under different conditions. After 7 days in culture, we harvested cells and measured the expression of Hes1 by quantitative RTPCR. hD1R up-regulated Hes1 expression in the hematopoietic cells in the co-culture with HUVECs + h5GF compared to other groups. Moreover, GSI effectively blocked Hes1 expression (Additional file 1: Figure S1C). These results suggested that live HUVECs were necessary for hD1R to activate Notch signaling. Next, we observed that hD1R had the effect on CD34+ ${ }^{+}$cells in different culture conditions. Seven days after the starting of the coculture, we found that total cell numbers significantly increased in the culture with HUVECs supplemented with $\mathrm{h} 5 \mathrm{GF}$ in the presence of $\mathrm{hD} 1 \mathrm{R}$, resulting in a fold expansion of $123.5 \pm 3.9$ nucleated cells (Fig. 1a; Additional file 1: Figure S2). FACS analysis showed that the percentage of $\mathrm{CD}_{3} 4^{+}$cells increased significantly in the culture with HUVECs plus hD1R plus h5GF (Fig. 1b, d), and the number of CD34 ${ }^{+}$cells was expanded $87.5 \pm 2.5-$ folds. Supernatant containing soluble angiocrine factors from HUVECs failed to efficiently expand CD34 ${ }^{+}$cells and accelerated $\mathrm{CD}_{4}{ }^{+}$cells differentiation (Fig. 1c, d). Similarly, on co-culture with fixed HUVECs, total cells number and $\mathrm{CD} 34^{+}$cells number significantly decreased. The expanded cells from each group were subjected to CFU assay. The results showed that the total number of colonies and the numbers of burst-forming unit-erythroid (BFU-E), CFU-granulocyte macrophage (CFU-GM), and CFU-granulocyte, erythrocyte, monocyte, macrophage (CFU-GEMM) significantly increased in the coculture with HUVECs in the presence of hD1R plus h5GF (Fig. 1e, f). Thus, these data showed that expansion of
HSPCs in the presence of hD1R required live HUVECs and direct cell-cell contacts between hematopoietic cells and HUVECs.

We analyzed cell apoptosis in the ex vivo culture by using annexin $\mathrm{V}$ staining followed by FACS. The data showed that a onefold less portion of $\mathrm{CD} 34^{+}$underwent apoptosis (defined as annexin $\mathrm{V}$ positive, propidium iodide negative $\mathrm{CD}^{+} 4^{+}$cells by FACS) in the co-culture with HUVECs in the presence of hD1R. However, coculture without HUVECs or with fixed HUVECs promoted cell apoptosis (Fig. 2a; Additional file 1: Figure S3). These data suggested that hD1R provided a survival signal and inhibited apoptosis in the co-culture with live HUVECs. Similarly, cell-cycle analysis indicated CD $34^{+}$ cells expanded on HUVECs with hD1R + h5GF maintained higher G0/G1 phases than those co-cultured without HUVECs or with fixed HUVECs (Fig. 2b, c), which prevented exhaustion of cycling CD34 ${ }^{+}$cells.

\section{hD1R promoted long-term repopulating capacity of HSPCs} To assess functional HSCs expanded by hD1R, we quantified SRC frequency by limiting-dilution analysis. Different numbers $\left(10^{3}, 6 \times 10^{3}, 10 \times 10^{3}\right.$ starting cells) of freshly isolated CD34 ${ }^{+}$cells (Day 0) or their progeny expanded on HUVECs + h5GF in the presence of PBS or hD1R were transplanted into sublethally irradiated NOD/SCID mice. The percentages of $\mathrm{hCD} 45^{+}$cells in peripheral blood were determined by FACS 12 weeks later. The results showed a 3.6-folds higher of the frequency of repopulating cells in the hD1R-expanded population $(1 / 5765$, with a $95 \%$ confidence interval from $1 / 7805$ to $1 / 4258$ ) than that in the PBS group $(1 / 21025$, with a $95 \%$ confidence interval from $1 / 32613$ to $1 / 13555)$, and a 8.1 -folds increase compared to that in the Day 0 group (1/46455, with a $95 \%$ confidence interval from $1 / 86190$ to $1 / 25039$ ) (Fig. 3a). This represented a total SRC content in three independent experiments (Additional file 1: Table S1). In the $10 \times 10^{3}$ cell groups, the cells cultured in the presence of $\mathrm{hD} 1 \mathrm{R}$ exhibited significantly higher level of engraftment than the cells of the control (Fig. 3b, c). To confirm that hD1R expanded HSCs with serial repopulating capacity, we performed a second engraftment experiment for the recipient mice in the $10 \times 10^{3}$ cells group. We infused $50 \%$ bone marrow of primary recipients of hD1R-expanded population or PBS-expanded ones into secondary NOD/SCID mice. The percentages of engrafted mice (with $\geq 0.5 \%$ hCD $45^{+}$ cells in BM) were analyzed by FACS 12 weeks post transplantation. The mean engraftment level in the recipients of hD1R-expanded group was higher than that in the PBS-expanded one (Fig. 3d). These data indicated that hD1R with HUVECs plus h5GF promoted a substantial increase of long-term repopulating HSCs in culture. 

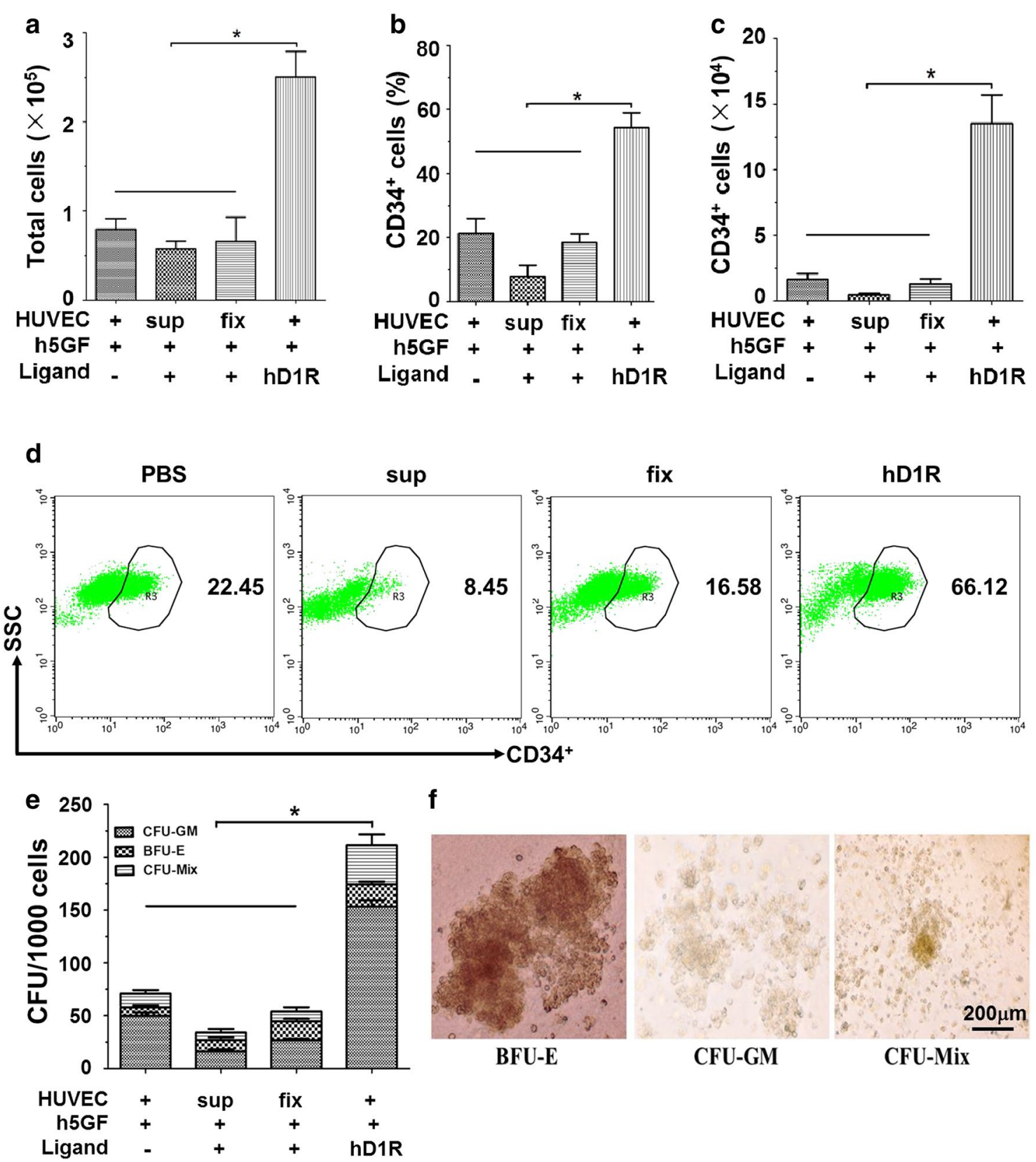

f

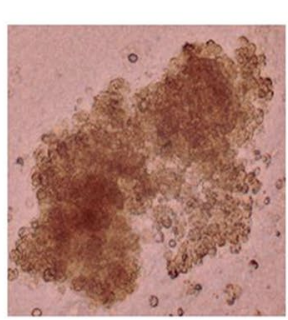

BFU-E

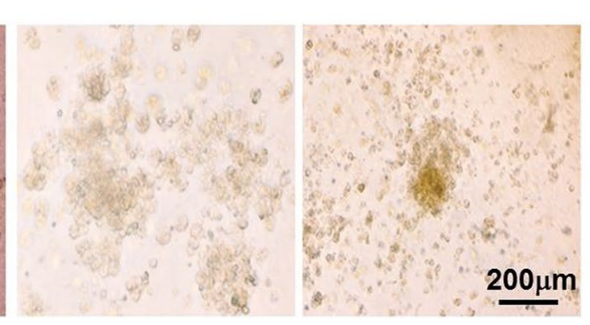

CFU-GM
CFU-Mix

Fig. 1 Human UCB CD34+ cells expansion by hD1R requires direct cellular interaction with live HUVECs. a CD34 ${ }^{+}$cells $\left(2 \times 10^{3}\right)$ were cultured with or without HUVECs in the presence of h5GF plus hD1R. The cells were harvested on day 7 and counted $(n=4)$. $\mathbf{b}, \mathbf{c}$ The cells in (a) were analyzed by FACS to determine the percentage $(\mathbf{b})$ and the number $(\mathbf{c})$ of CD34 $4^{+}$cells $(n=4)$. d Representative FACS analysis of CD34 ${ }^{+}$cells in $(\mathbf{b})$. e The cells $\left(1 \times 10^{3}\right)$ in $(\mathbf{a})$ were used for colony-forming assay $(n=4)$. f Photomicrographs of human hemopoietic cell colonies. Scale bar represented $200 \mu \mathrm{m}$. Bars $=$ mean $\pm S D$. ${ }^{*} P<0.05$

\section{hD1R enhanced HSC homing to the marrow}

Increased HSC frequency by hD1R could result from HSC number increase or cell-cycle status or improved homing capacity of HSCs. To evaluate the homing efficiency of $\mathrm{CD} 34^{+}$cells, we transplanted cultured CD $34^{+}$ cells derived from different conditions into sublethally irradiated NOD/SCID mice. There was no difference in the percentage of $\mathrm{hCD} 45^{+}$cells homing to marrow between PBS-cultured cells and Day 0 cells. However, significantly more $\mathrm{hCD} 45^{+}$cells homing to the marrow in hD1R-cultured ones (Fig. 4a). Numerous cooperative mechanisms were believed to regulate HSC homing to the marrow, which included adhesion receptor molecules, the stromal cell-derived factor-1 (SDF-1)-CXCR4 

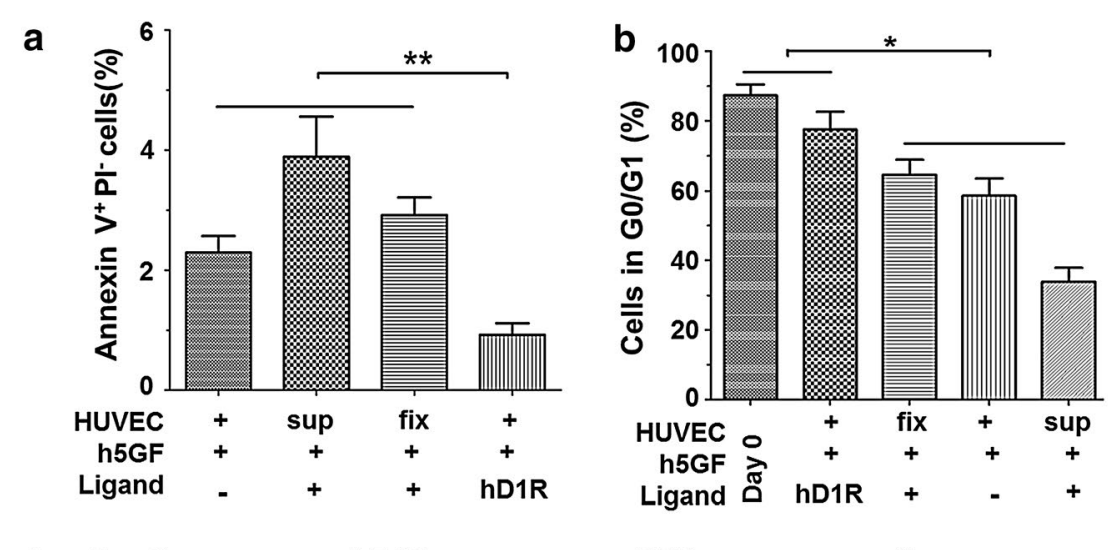

C Day 0

hD1R

PBS

fix

sup

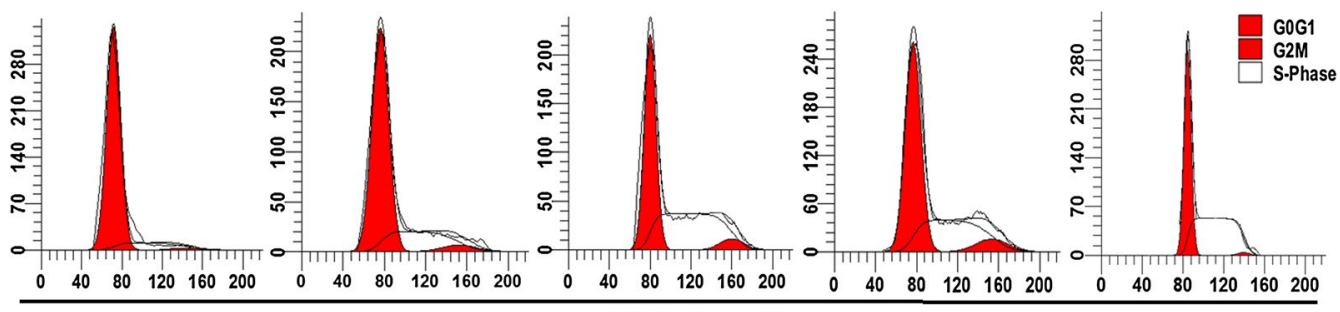

PI intensity

Fig. 2 hD1R maintained quiescence and regeneration of human UCB CD34 ${ }^{+}$cells. CD34 ${ }^{+}$cells were cultured with or without HUVECs in the presence of h5GF plus hD1R. The cells were harvested on day 7. a Cultured cells were analyzed by using annexin $V$ staining followed by FACS to determine the percentage of cell apoptosis $(n=4)$. $\mathbf{b}$ Cell-cycle analysis of $\mathrm{CD}_{3} 4^{+}$cells expanded in different conditions or freshly purified from UCB (Day 0). The number of cells in various stages of the cell cycle was quantified measuring the area under the red peaks (G0/G1). c Representative plots of cell-cycle analysis of $\mathrm{CD}_{4}{ }^{+}$cells in $(\mathbf{c})$. Bars $=$ mean $\pm \mathrm{SD} .{ }^{*} P<0.05$

and so on. To determine whether the enhanced homing of hD1R-cultured cells was due to up-regulation of SDF-1/CXCR4 signaling, we performed in vitro migration assays and found a significant increase in hD1R-cultured cells migration toward SDF-1 (Fig. 4b). In addition, we tested expression levels of some adhesion receptor molecules and CXCR4 in the cultured cells. The result showed hD1R did not up-regulate CXCR4 expression on the cultured cells, but increased $\alpha 4$ and $\alpha 6$ integrin mRNA levels (Fig. 4c).

\section{hD1 R promoted donor cell engraftment in BM transplantation}

We assessed whether hD1R administration could augment the proliferation of transplanted donor cells in the host. Sublethally irradiated NOD/SCID mice were transfused with the limiting dose of human UCB CD34 ${ }^{+}$cells $\left(5 \times 10^{4}\right.$ cells), and were injected i.p. daily with PBS or $\mathrm{hD1R}(50 \mu \mathrm{g})$ from the day of BM transplantation for 7 days. On day 14 after irradiation, histological examination of BM showed that the structure of BM from the $\mathrm{hD1R}$ group showed significantly increased cellularity compared to the PBS or control group (irradiated mice receiving no donor cells) (Fig. 5a). The number of total bone marrow cells in hD1R-treated mice was higher than those in the PBS group $\left(7.09 \pm 0.26 \times 10^{6}\right.$ vs. $2.54 \pm 0.16 \times 10^{6}, P<0.01$, Fig. $\left.5 \mathrm{~b}\right)$. At 4 weeks after transplantation, the percentages of donor cells (hCD45 cells) in peripheral blood were determined by FACS. The hD1R-treated mice significantly increased $\mathrm{hCD} 45^{+}$cell repopulation compared with the PBS group (Fig. 5c, d). Colony-forming assay of BM cells showed higher BM CFU counts in hD1R-treated mice than in PBS-treated ones (Fig. 5e). These results showed that hD1R promoted human CB HSPCs regeneration in vivo and accelerated hematopoietic recovery, particularly in a setting where the HSC dose was limiting.

\section{Discussion}

We previously demonstrated a new type of soluble Notch ligand composed of the DSL domain of Dll1 and a RGD motif (D1R) had a strong activity to activate Notch signaling and enhanced HSPCs expansion ex vivo [25, 26]. Herein, we evaluated the in vivo effect of $\mathrm{hD} 1 \mathrm{R}$ on hematologic recovery and the engraftment of donor cells in HSCT. 

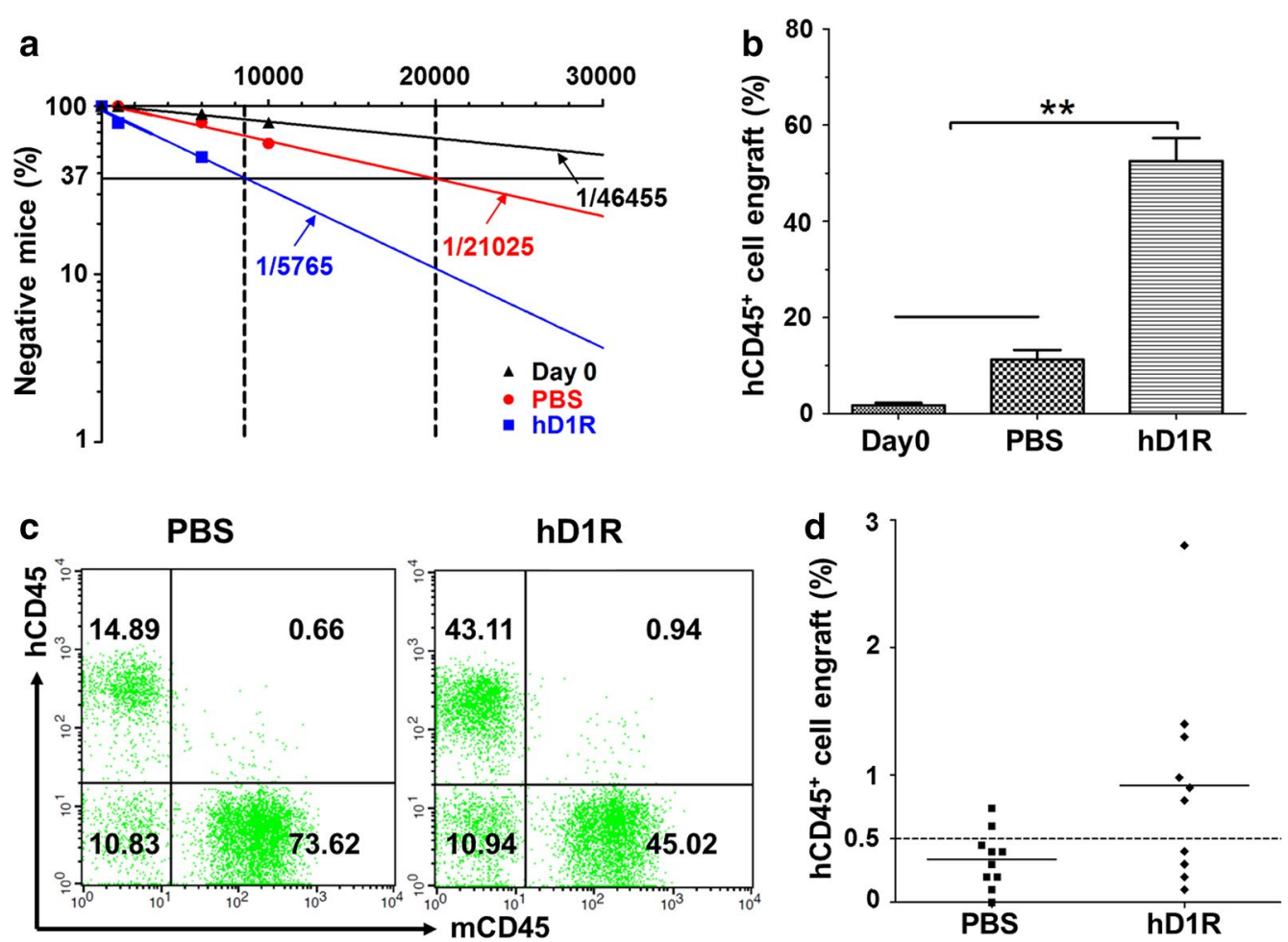

Fig. 3 Repopulation of sublethally irradiated mice with expanded human $\mathrm{CD}_{3} 4^{+}$cells. a SRC assay. CD $34^{+}$cells freshly purified from UCB (Day 0) $\left(10^{3}, 6 \times 10^{3}, 10 \times 10^{3}\right.$ starting cells) or their progeny expanded with HUVECs + h5GF plus PBS or hD1R were transplanted into sublethally irradiated NOD/SCID mice. The peripheral blood cells of the recipients were analyzed by FACS 12 weeks post transplantation. The number of transplanted cells was plotted against the percentage of mice without engraftment to determine the frequency of repopulating units $(n=30)$. $\mathbf{b}$, c The cells in (a) were transfused into sublethally irradiated NOD-SCID mice. In the $10 \times 10^{3}$ cells group, the peripheral blood cells of the recipients were analyzed by FACS 12 weeks post transplantation. The percentages of engrafted human CD45 were compared $(\mathbf{b})(n=10)$. c Representative FACS analysis of hCD $45^{+}$cell engraftment in (b). d Human $\mathrm{CD} 45^{+}$cell engraftment 12 weeks after the secondary transplantation for primary recipient mice in the $10 \times 10^{3}$ cells group. Horizontal bars represented mean levels of $\mathrm{hCD} 45^{+}$cell engraftment in $\mathrm{BM}$. $(\mathrm{n}=10)$. Bars $=$ mean $\pm \mathrm{SD}$. ${ }^{*} P<0.05$

We found that hD1R supplemented HUVEC monolayers and a cytokine combination significantly decreased cell apoptosis and induced UCB HSPCs expansion, which were consistent with our previous study [25]. In addition, exposure to $\mathrm{h} 5 \mathrm{GF}$ plus $\mathrm{hD1R}$ plus supernatant in the absence of HUEVCs or fixed HUVECs caused a decline in HSPCs. Moreover, hD1R in combination with h5GF plus live HUVECs was able to maintain higher G0/ G1 populations of HSPCs, which were capable of rapid multi-lineage reconstitution and maintain long-term SCID-repopulating capacity in serial transplantation. Delaney et al. reported a Delta $1^{\text {ext-IgG }}$-mediated ex vivo expansion system for human UCB HSPCs which resulted in a marked increase of HSPCs. When these expanded cells were infused to patients undergoing a myeloablative preparative regimen, the time to neutrophil recovery was significantly reduced. However, longer-term engraftment of donor cells derived from the expanded cells was observed in one of ten patients, suggesting a deficiency of true HSCs expansion and that early myeloid recovery was attributed to short-term repopulating progenitor cells. Of course, we could not exclude this possibility that the expanded cells was rejected by the unmanipulated cells [24]. The function of HSCs might be impaired because the ex vivo culture system deprived HSCs of microenvironmental components [27]. Previous studies demonstrated the vascular niche regulates the survival, proliferation and differentiation of HSCs. Bone marrow sinusoidal ECs played functionally important roles [28]. Butler et al. [13] confirmed the role of the ECs was essential in HSC expansion. Similarly, our results demonstrated live HUVECs were necessary for HSPCs expansion. Moreover, hD1R may promote closer cellular contact between HSCs and HUVECs, which mimiced a physiological microenvironment to improve self-renewal and proliferation of HSCs.

Previous study demonstrated mD1R could directly stimulate hematopoiesis in vivo in normal mice and sublethally irradiated mice [25]. Here, we found that hD1R administration dramatically enhanced the engraftment 

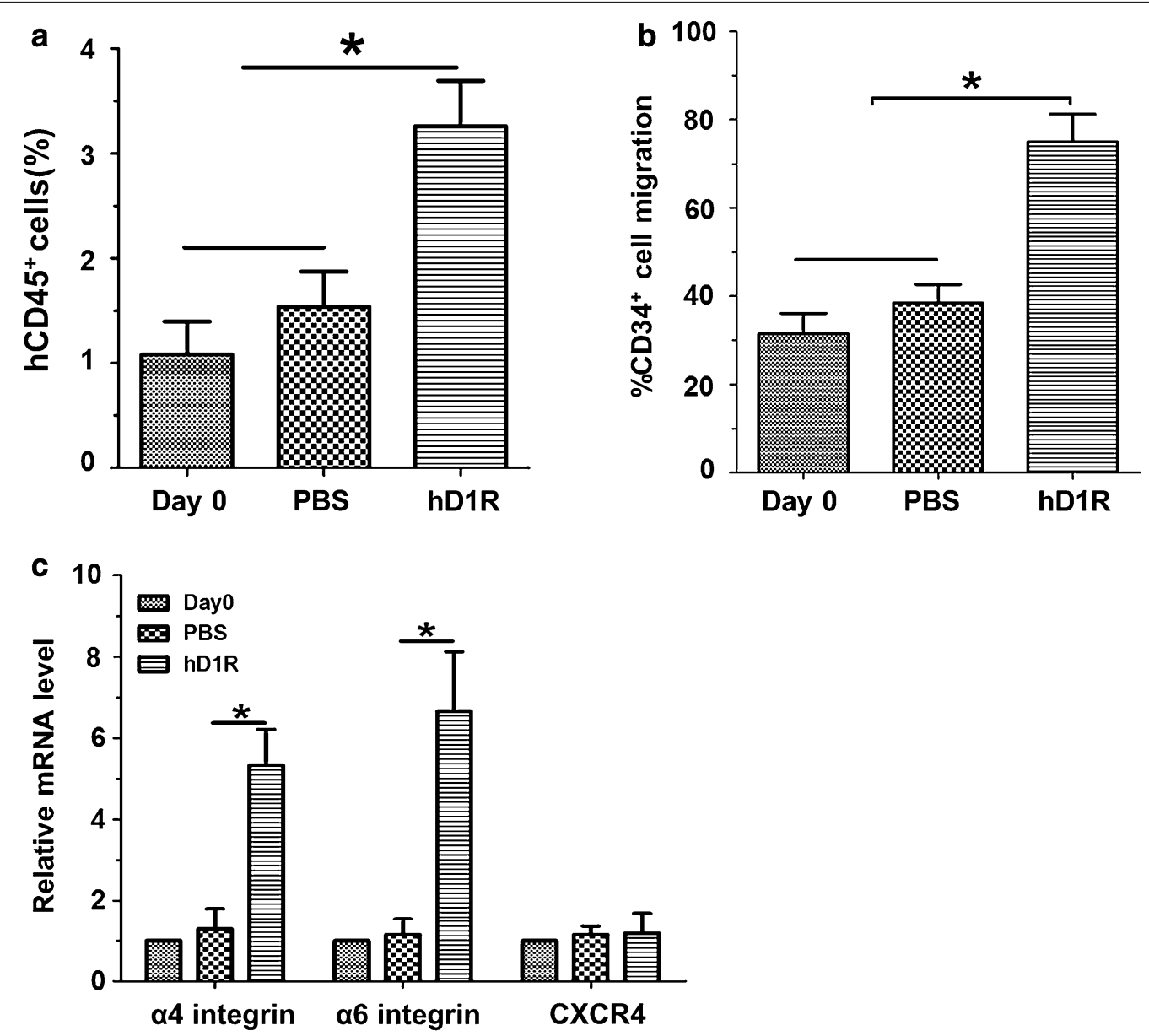

Fig. 4 hD1R enhanced the homing efficiency of CD34 ${ }^{+}$cells. a Homing efficiency. CD34 ${ }^{+}$cells $\left(2 \times 10^{5}\right)$ were purified from UCB (Day 0$)$ or cells expanded ex vivo transplanted into sublethally irradiated mice. BM cells from the recipients were analyzed by FACS $16 \mathrm{~h}$ after the transplantation $(n=8)$. b Migration assay. Freshly purified CD34 cells or cells $\left(2 \times 10^{5}\right)$ expanded ex vivo were cultured in the upper compartment of transwell for $4 \mathrm{~h}$. Total cell migration was quantitated by flow cytometry. Migrated cells toward SDF-1 in the lower chamber significantly increased in hD1Rcultured ones $(n=4)$. c hD1R increased the expression of integrins in CD34 ${ }^{+}$cells. Human CD34 ${ }^{+}$cells were incubated with HUVECs + h5GF in the presence of PBS or hD1R for 7 days. The hematopoietic cells in suspension or Day 0 CD34 ${ }^{+}$cells were collected, and the expression of a4, a6 integrins, and CXCR4 was estimated by using real time RT-PCR $(n=4)$. Bars $=$ mean $\pm S D$. ${ }^{*} P<0.05$

of donor HSCs after transplantation. Moreover, hD1R significantly boosted the proliferation of BM donor cells and promoted the recovery of the structure and organization of BM microenvironment after irradiation, consistent with the role of Notch signaling in regulating EC proliferation and differentiation [29]. Meanwhile, we continuously monitored the mice injected with hD1R for body weight and hematopoietic parameters until the end of the study. We did not observe tumors occurred, suggesting hD1R may be safe for further application. Many clinical studies showed that the doses of total nucleated cells and $\mathrm{CD} 34^{+}$cells in UCBT affected the rate of neutrophil and platelet engraftment, which was closely related with the incidence of transplant-related complication and overall survival [30]. We also found that hD1R substantially shortened the average time of neutrophil and platelet recovery (data not shown). These results demonstrated that $\mathrm{hD1R}$ may have a potential to be used in clinical therapy of human diseases such as myelotoxic chemo- or radio-therapy and increase the efficiency of UCBT.

It was reasonable that the rapid engraftment was likely a result of the direct expansion of HSPCs ex vivo and in vivo by hD1R. Moreover, hD1R improved homing capacity of HSCs to facilitate UCB HSPCs engraftment. Studies showed Notch signaling regulated the expression of CXCR4, a critical receptor for HSC homing [31, 32]. Here, hD1R didn't up-regulate CXCR4 expression on HSPCs. Indeed, the mRNA levels of the $\alpha 4$ and $\alpha 6$ integrin were up-regulated, consistent with the role of 


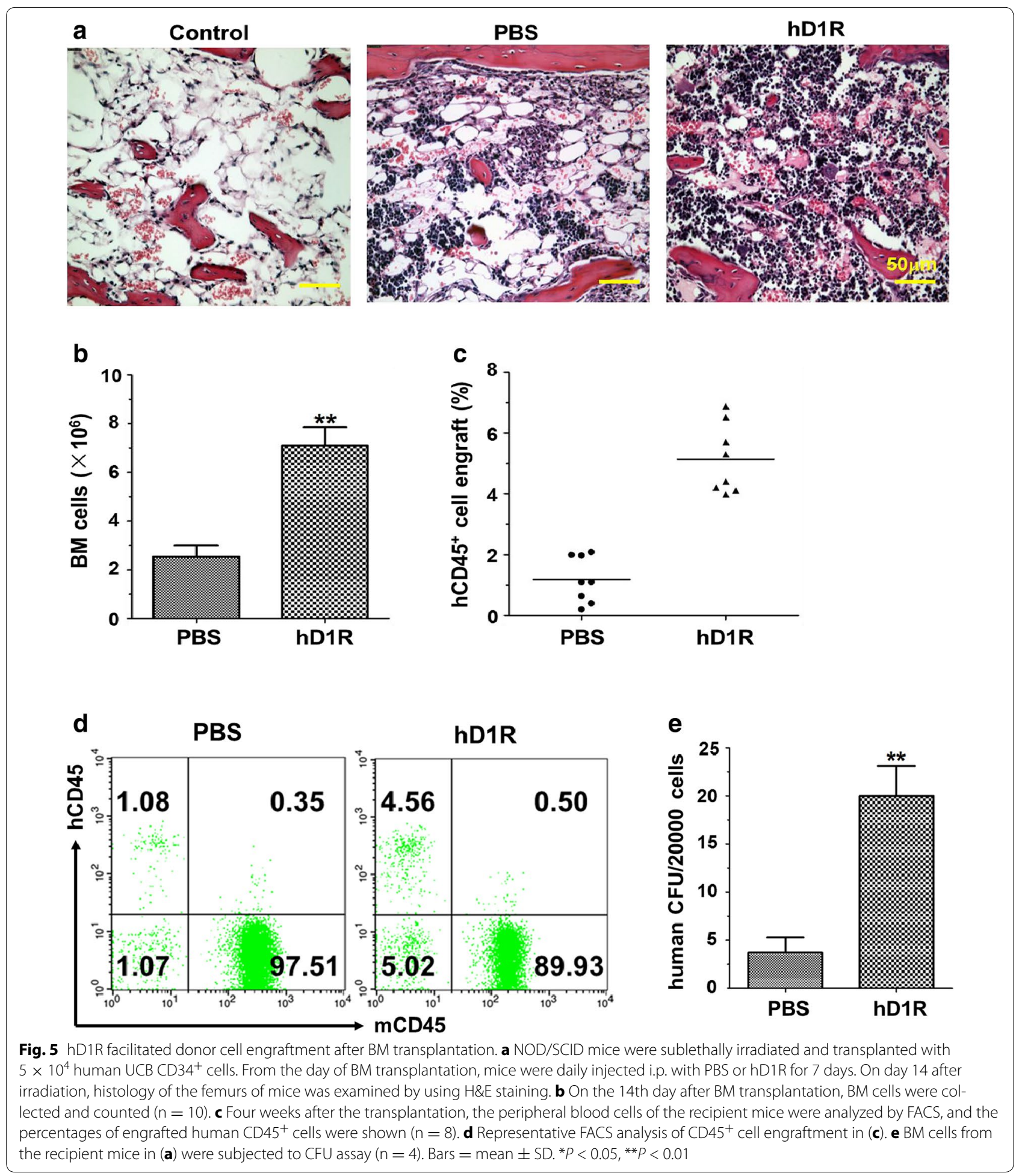

adhesion molecules on transplanted HSCs in increasing engraftment [33, 34]. It was possible that Notch signaling crosstalked with other important signaling pathways involved in stem cell niches to help the homing and proliferation of HSCs [35].

\section{Conclusions}

In summary, this study showed that hD1R promoted both hematopoietic recovery and long-term engraftment of human UCB HSPCs. hD1R may represent a new cytokine and have potential clinical utility for HSCT. 


\section{Additional file}

Additional file 1. Human UCB CD34+ cells expansion by $\mathrm{hD} 1 \mathrm{R}$ requires direct cellular interaction with live HUVECs.

\section{Abbreviations}

UCB: umbilical cord blood; HSPC: hematopoietic stem and progenitor cell; DSL: Delta-Serrate-Lag-2; HUVEC: human umbilical vein endothelial cell; LT-HSC: long-term hematopoietic stem cell; NOD/SCID: Nonobese DiabeticSevere Combined Immunodeficiency; Hes: Hairy and enhancer of split; BM: bone marrow; LB: Luria-Bertani; IPTG: isopropyl $\beta$-D-thiogalactoside; FBS: fetal bovine serum; ECGS: endothelial cell growth supplement; PFA: paraformaldehyde; CFU: Colony-forming units; BFU-E: burst-forming unit-erythroid; CFUGM: CFU-granulocyte macrophage; CFU-GEMM: CFU-granulocyte, erythrocyte, monocyte, macrophage; SRC: SCID-repopulating cell; PB: peripheral blood.

\section{Authors' contributions}

YML and YQZ designed the study. DMT performed the laboratory work for this study and analyzed data. DMT wrote the paper. YML and YQZ revised and approved the final manuscript. All authors read and approved the final manuscript.

\section{Author details}

${ }^{1}$ Department of Hematology, 309th Hospital, Chinese People's Liberation Army, Hei-san hu Street \#17, 100091 Beijing, China. ${ }^{2}$ Department of Hematology, Tangdu Hospital, Fourth Military Medical University, Xi'an, China.

\section{Acknowledgements}

This work was supported by grants from the Ministry of Science and Technology (2009CB521706, 2011ZXJ09101-02C, 2009ZX09301-009-RC11, CWS1 1 J143), the Natural Science Foundation of China (30900496, 81400075) and 309th Hospital (2014ZD-002). This study was implemented in the Graduates Innovation Center of Fourth Military Medical University.

\section{Competing interests}

The authors declare that they have no competing interests.

Received: 10 August 2015 Accepted: 26 December 2015

Published online: 06 January 2016

\section{References}

1. Kurtzberg J, Laughlin M, Graham ML, Smith C, Olson JF, Halperin EC, et al. Placental blood as a source of hematopoietic stem cells for transplantation into unrelated recipients. N Engl J Med. 1996;335(3):157-66.

2. Sorrentino BP. Clinical strategies for expansion of haematopoietic stem cells. Nat Rev Immunol. 2004;4(11):878-88.

3. Kelly SS, Parmar S, De Lima M, Robinson S, Shpall E. Overcoming the barriers to umbilical cord blood transplantation. Cytotherapy. 2010;12(2):121-30.

4. Delaney C, Ratajczak MZ, Laughlin MJ. Strategies to enhance umbilical cord blood stem cell engraftment in adult patients. Expert Rev Hematol. 2010;3(3):273-83.

5. Dahlberg A, Delaney C, Bernstein ID. Ex vivo expansion of human hematopoietic stem and progenitor cells. Blood. 2011;117(23):6083-90.

6. Wagner JE Jr, Eapen M, Carter S, Wang Y, Schultz KR, Wall DA, et al. Oneunit versus two-unit cord-blood transplantation for hematologic cancers. N Engl J Med. 2014;371(18):1685-94.

7. Von Drygalski A, Alespeiti G, Ren L, Adamson JW. Murine bone marrow cells cultured ex vivo in the presence of multiple cytokine combinations lose radioprotective and long-term engraftment potential. Stem Cells Dev. 2004;13(1):101-11.

8. Walasek MA, van Os R, de Haan G. Hematopoietic stem cell expansion: challenges and opportunities. Ann NY Acad Sci. 2012;1266:138-50.

9. Wilson A, Trumpp A. Bone-marrow haematopoietic-stem-cell niches. Nat Rev Immunol. 2006;6(2):93-106.
10. Morrison SJ, Spradling AC. Stem cells and niches: mechanisms that promote stem cell maintenance throughout life. Cell. 2008;132(4):598-611.

11. Blank U, Karlsson G, Karlsson S. Signaling pathways governing stem-cell fate. Blood. 2008;111(2):492-503.

12. Cook MM, Doran MR, Kollar K, Barbier V, Winkler IG, Levesque JP, et al. Engraftment outcomes after HPC co-culture with mesenchymal stromal cells and osteoblasts. J Clin Med. 2013;2(3):115-35.

13. Butler JM, Nolan DJ, Vertes EL, Varnum-Finney B, Kobayashi H, Hooper AT, et al. Endothelial cells are essential for the self-renewal and repopulation of Notch-dependent hematopoietic stem cells. Cell Stem Cell. 2010;6(3):251-64

14. Corselli M, Chin CJ, Parekh C, Sahaghian A, Wang W, Ge S, et al. Perivascular support of human hematopoietic stem/progenitor cells. Blood. 2013;121(15):2891-901.

15. Bigas A, Espinosa L. Hematopoietic stem cells: to be or Notch to be. Blood. 2012;119(14):3226-35.

16. Pajcini KV, Speck NA, Pear WS. Notch signaling in mammalian hematopoietic stem cells. Leukemia. 2011;25(10):1525-32.

17. Walker L, Carlson A, Tan-Pertel HT, Weinmaster G, Gasson J. The notch receptor and its ligands are selectively expressed during hematopoietic development in the mouse. Stem Cells. 2001;19(6):543-52.

18. Singh N, Phillips RA, Iscove NN, Egan SE. Expression of notch receptors, notch ligands, and fringe genes in hematopoiesis. Exp Hematol. 2000;28(5):527-34.

19. Stier S, Cheng T, Dombkowski D, Carlesso N, Scadden DT. Notch1 activation increases hematopoietic stem cell self-renewal in vivo and favors lymphoid over myeloid lineage outcome. Blood. 2002;99(7):2369-78.

20. Csaszar E, Wang W, Usenko T, Qiao W, Delaney C, Bernstein ID, et al. Blood stem cell fate regulation by Delta-1-mediated rewiring of IL-6 paracrine signaling. Blood. 2014;123(5):650-8.

21. Vas V, Szilágyi L, Pálóczi K, Uher F. Soluble Jagged-1 is able to inhibit the function of its multivalent form to induce hematopoietic stem cell selfrenewal in a surrogate in vitro assay. J Leukoc Biol. 2004;75(4):714-20.

22. Varnum-Finney B, Wu L, Yu M, Brashem-Stein C, Staats S, Flowers D, et al. Immobilization of Notch ligand, Delta-1, is required for induction of notch signaling. J Cell Sci. 2000;113(Pt 23):4313-8.

23. Delaney C, Varnum-Finney B, Aoyama K, Brashem-Stein C, Bernstein ID. Dose-dependent effects of the Notch ligand Delta1 on ex vivo differentiation and in vivo marrow repopulating ability of cord blood cells. Blood. 2005;106(8):2693-9.

24. Delaney C, Heimfeld S, Brashem-Stein C, Voorhies H, Manger RL, Bernstein ID. Notch-mediated expansion of human cord blood progenitor cells capable of rapid myeloid reconstitution. Nat Med. 2010;16(2):232-6.

25. Tian DM, Liang L, Zhao XC, Zheng MH, Cao XL, Qin HY, et al. Endotheliumtargeted Delta-like 1 promotes hematopoietic stem cell expansion ex vivo and engraftment in hematopoietic tissues in vivo. Stem Cell Res. 2013;11(2):693-706.

26. Zhao XC, Dou GR, Wang L, Liang L, Tian DM, Cao XL, et al. Inhibition of tumor angiogenesis and tumor growth by the DSL domain of human Delta-like 1 targeted to vascular endothelial cells. Neoplasia. 2013;15(7):815-25.

27. Mayani $\mathrm{H}$. Notch signaling: from stem cell expansion to improving cord blood transplantation. Expert Rev Hematol. 2010;3(4):401-4.

28. Hooper AT, Butler JM, Nolan DJ, Kranz A, lida K, Kobayashi M, et al. Engraftment and reconstitution of hematopoiesis is dependent on VEGFR2-mediated regeneration of sinusoidal endothelial cells. Cell Stem Cell. 2009;4(3):263-74.

29. Dou GR, Wang YC, Hu XB, Hou LH, Wang CM, Xu JF, et al. RBP-J, the transcription factor downstream of Notch receptors, is essential for the maintenance of vascular homeostasis in adult mice. FASEB J. 2008;22(5):1606-17

30. Wagner JE, Barker JN, DeFor TE, Baker KS, Blazar BR, Eide C, et al. Transplantation of unrelated donor umbilical cord blood in 102 patients with malignant and nonmalignant diseases: influence of CD34 cell dose and HLA disparity on treatment-related mortality and survival. Blood. 2002;100(5):1611-8.

31. Majka M, Ratajczak MZ. Biological role of the CXCR4-SDF-1 axis in normal human hematopoietic cells. Methods Mol Biol. 2006;332:103-14.

32. Wang L, Wang YC, Hu XB, Zhang BF, Dou GR, He F, et al. Notch-RBP-J signaling regulates the mobilization and function of endothelial progenitor 
cells by dynamic modulation of CXCR4 expression in mice. PLoS One. 2009;4(10):e7572.

33. Qian H, Tryggvason K, Jacobsen SE, Ekblom M. Contribution of a6 integrins to hematopoietic stem and progenitor cell homing to bone marrow and collaboration with a4 integrins. Blood. 2006;107(9):3503-10.
34. Hartz B, Volkmann T, Irle S, Loechelt C, Neubauer A, Brendel C. a4 integrin levels on mobilized peripheral blood stem cells predict rapidity of engraftment in patients receiving autologous stem cell transplantation. Blood. 2011;118(8):2362-5.

35. Blank U, Karlsson G, Karlsson S. Signaling pathways governing stem-cell fate. Blood. 2008;111(2):492-503.
Submit your next manuscript to BioMed Central and we will help you at every step:

- We accept pre-submission inquiries

- Our selector tool helps you to find the most relevant journal

- We provide round the clock customer support

- Convenient online submission

- Thorough peer review

- Inclusion in PubMed and all major indexing services

- Maximum visibility for your research

Submit your manuscript at www.biomedcentral.com/submit
() Biomed Central 